\title{
Produção de massa de tomate com maior conteúdo de pró-vitamina A: avaliação microbiológica, físico-química e sensorial
}

\author{
Mariana Silva Pelosi ${ }^{2}$, Cristiane Hess de Azevedo-Meleiro ${ }^{3}$ \\ http://dx.doi.org/10.1590/0034-737X201461060002
}

\section{RESUMO}

A deficiência de vitamina A é um grande problema de saúde pública e um dos grupos mais vulneráveis a essa deficiência são as crianças em idade pré-escolar. Existem medidas de intervenção que podem ser adotadas como forma de preveni-la ou reduzi-la, sendo uma delas o enriquecimento de alimentos. O objetivo deste trabalho foi produzir massa de tomate com fontes naturais de carotenoides pró-vitamínicos A, oriundos de três diferentes concentrações de suco de cenoura, e caracterizar as formulações em relação à análise microbiológica (bactérias lácticas, bolores e leveduras, clostrídios butíricos e termófilos "flat-sour"), análise físico-química (pH, sólidos solúveis e carotenoides) e análise sensorial (teste de aceitação com escala hedônica e intenção de compra). Os resultados mostraram que todas as formulações foram aprovadas no teste microbiológico, durante 90 dias de armazenamento. Quanto às análises físico-químicas, verificou-se aumento significativo do $\mathrm{pH}$, nas três formulações, enquanto o teor de sólidos solúveis manteve-se inalterado, durante os três meses, em cada um dos produtos. Apesar da degradação significativa ao longo do tempo, foi possível aumentar o conteúdo de carotenoides nas massas de tomate com suco de cenoura, nas formulações. Além disso, as formulações foram consideradas, sensorialmente, como boas, pelos provadores. Considerando-se o teor de carotenoides encontrados e a impressão sensorial, as formulações podem ser uma estratégia de prevenção ou de redução da carência de vitamina A.

Palavras-chave: saúde, carotenoides, vitamina A.

\section{ABSTRACT}

\section{Production of tomato paste with higher pro-vitamin A content: microbiological, physico-chemical and sensorial evaluation}

Vitamin A deficiency is an important public health problem, and one of the most susceptible human groups to this deficiency are children at preschool age. There are intervention measures that can be adopted as a way to prevent or reduce this disease, and food fortification is one of them. The objectives of this study were to produce a tomato paste with natural sources of pro-vitamin A carotenoids from different concentrations of carrot juice, characterize these products in relation to microbiological security (lactic acid bacteria, yeasts and molds, butyric clostridia and thermophilic "flat-sour"), physic-chemical analysis (pH, soluble solids and carotenoids) and sensorial analysis (hedonic scale with acceptance test and purchase intent). The results showed that all the formulations were approved in the microbiological test during 90 days of storage. The physical-chemical analysis showed a significant increase in the $\mathrm{pH}$ of the three formulations, while the soluble solids remained unchanged during the

Recebido para publicação em 14/09/2012 e aprovado em 13/10/2014

'Este trabalho foi extraído da dissertação de Mestrado da primeira autora. Fonte financiadora: Capes.

${ }^{2}$ Nutricionista, Mestre. Departamento de Tecnologia de Alimentos, Universidade Federal Rural do Rio de Janeiro, Rodovia BR 465, km 7, 23890-000, Seropédica, Rio de Janeiro, Brasil.mariana_pelosi@yahoo.com.br (Autora para correspondência)

${ }^{3}$ Engenheira de Alimentos, Doutora. Departamento de Tecnologia de Alimentos, Universidade Federal Rural do Rio de Janeiro, Rodovia BR 465, km 7, 23890-000, Seropédica, Rio de Janeiro, Brasil. hesscris@yahoo.com.br 
three months in each of these products. Despite the significant degradation over time, the increase in the content of carotenoids in the tomato paste with carrot juice was possible in these formulations. Moreover, these formulations were characterized as good by sensorial assessors. Considering the content of carotenoids found and sensorial impression, these formulations can be a strategy to prevent or reduce the vitamin A deficiency.

Key words: health, carotenoids, vitamin A.

\section{INTRODUÇÃO}

Em 2009, a Organização Mundial da Saúde (OMS) estimou que há, no mundo, 5,2 milhões de crianças em idade pré-escolar com xeroftalmia e 190 milhões de crianças, também em idade pré-escolar, com concentração sérica de retinol abaixo de $0,70 \mu \mathrm{mol} / \mathrm{L}$.

No Brasil, a deficiência encontra-se em vários estados e capitais, em cidades grandes e pequenas e em zonas rurais. Inquéritos bioquímicos disponíveis confirmam que a carência de vitamina Aé um problema de saúde pública nos estados de São Paulo, Minas Gerais, Pernambuco, Paraíba, Ceará, Bahia e Amazonas (Ramalho et al. 2002; Geraldo et al. 2003).

Considerando-se a dimensão coletiva da hipovitaminose A, refletida nas taxas de mortalidade do grupo materno-infantil do país, estudos apontam para a necessidade de incluir estratégias de intervenção adicionais à suplementação, que podem ser adotadas para prevenir ou reduzir a deficiência de vitamina A, contribuindo para a reversão desse quadro. Dentre essas medidas, pode-se citar a educação nutricional e a formulação de produtos com alto conteúdo da vitamina (OMS, 2000; Milagres et al. 2007; Ramalho et al. 2008).

Atualmente, a legislação determina que, para vitaminas e minerais, o atributo fonte é dado ao produto sob condição mínima de haver 15\% da IDR (Ingestão Diária Recomendada) por $100 \mathrm{~g}$ ou $100 \mathrm{~mL}$, em pratos preparados, conforme o caso, ou por porção, e alto conteúdo, quando atingir no mínimo $30 \%$ da IDR, sob as mesmas condições anteriormente descritas. Pode, ainda, receber o atributo aumentado, quando ocorre aumento mínimo de $10 \%$ no conteúdo da vitamina ou mineral (Brasil, 2012).

Para obter-se aumento do conteúdo, faz-se importante a escolha do alimento a ser usado como veículo de transporte, pois o alimento pode interferir na absorção do composto, diminuindo sua biodisponibilidade. Dentre os fatores que podem afetar a biodisponibilidade dos carotenoides, pode-se citar a espécie do carotenoide, a natureza da matriz na qual os carotenoides estão incorporados, os efetuadores de absorção, as interações en- tre os carotenoides, o estado nutricional e os fatores genéticos (West, Castenmiller 1998).

Neste estudo, a vantagem do uso da massa de tomate como veículo de transporte é o fato de ser facilmente empregada em alimentos e ser, comumente, aceita por crianças, quando acrescentada em determinadas preparações. Ademais, estudos mostram que os produtos à base de tomate destacam-se pela presença de licopeno, o mais potente antioxidante dentre os carotenoides, entretanto, os mesmos não são considerados fonte de carotenoides pró-vitamínicos A (Stahl et al., 1998; Clinton, 1998).

Os carotenoides com ação pró-vitamínica A são, dentre aqueles de importância para a saúde humana, âcaroteno, $\alpha$-caroteno e $\beta$-criptoxantina (RodriguezAmaya, 1985). Tawata (2010) destacou a cenoura como uma das dez fontes mais ricas em $\alpha$-caroteno. Assim, sua adição, na forma de suco, ao produto à base de tomate, apresentou a intenção de incorporar carotenoides com tal atividade.

Além dos carotenoides que carreiam, os produtos possuem a vantagem de serem de origem vegetal e, apesar de não apresentarem vitamina A pré-formada, como em produtos de origem animal dependendo, assim, de fatores que afetarão a biodisponibilidade para a bioconversão, possivelmente apresentarão maior acesso pela população. Além disso, esse aumento no conteúdo não agrega fatores antinutricionais nem riscos de causar intoxicação por meio do consumo.

Em vista disso, o objetivo deste trabalho foi produzir massa de tomate com fonte natural de carotenoides pró-vitamínicos A, oriundo de três diferentes concentrações de suco de cenoura, e analisar as formulações em relação às características microbiológica, físicoquímica e sensorial.

\section{MATERIAL E MÉTODOS}

\section{Elaboração da massa de tomate}

Foi utilizado como matéria-prima o tomate (Solanum lycopersicum) var. "Carmen", que foi selecionado, limpado e sanitizado, com o uso de 50 ppm de 
cloro, durante 15 minutos de imersão. Em seguida, foi centrifugado em centrífuga Walita Juicer RI1858 Inox, para o aproveitamento do suco de tomate, excluindose, assim, casca e semente. O suco foi concentrado até aproximadamente $20{ }^{\circ}$ Brix, utilizando-se um evaporador rotativo a vácuo. Toda a massa de tomate foi produzida descontinuamente e armazenada à temperatura de $-18{ }^{\circ} \mathrm{C}$, até atingir a quantidade necessária para desenvolver os produtos e encaminhar para as diferentes análises. Quando a quantidade foi suficiente, a massa foi descongelada, em refrigeração. A partir daí, ingredientes como sacarose e sal foram incorporados ao concentrado, nas proporções de 1,2 e $0,2 \%$, respectivamente, permitindo, assim, a padronização da massa de tomate, que apenas se diferenciou de uma formulação para a outra com relação às proporções de suco de cenoura.

Para a obtenção do suco, a cenoura (Daucus carota) var. "Nantes" foi também lavada, sanitizada em água clorada a $50 \mathrm{ppm}$, por 15 minutos, cortada e o suco foi extraído na centrífuga descrita anteriormente, aproveitando-se a fração fluida. A adição do suco de cenoura à massa de tomate foi feita nas proporções de 20, 30 e $50 \%$. Após a realização das misturas, nas proporções necessárias, aqueceu-se cada formulação até $85^{\circ} \mathrm{C}$, para pasteurização. Em seguida, as formulações foram dispostas em embalagens de vidro com tampa metálica, previamente sanitizadas.

Após o envasamento, os potes foram invertidos, ou seja, dispostos em sentido contrário, permanecendo dessa forma por cinco minutos para esterilização da tampa. Em seguida, o resfriamento foi imediatamente realizado com água fria. Os potes foram armazenados para as análises, em estufa incubadora para estudo de prazo de validade, com temperatura regulada para $25^{\circ} \mathrm{C}$. A Figura 1 representa o fluxograma de execução do processo.

\section{Análise microbiológica}

As análises microbiológicas foram feitas no Laboratório Analítico de Alimentos e Bebidas, da Universidade Federal Rural do Rio de Janeiro (UFRRJ), no Instituto de Tecnologia (IT).

Considerando-se o produto comercialmente estéril e estável à temperatura ambiente, foi feito o teste de esterilidade comercial para produtos ácidos $(\mathrm{pH} \leq 4,5)$, conforme Brasil (2001). Para o teste, as amostras foram, inicialmente, pré-incubadas, a $30{ }^{\circ} \mathrm{C}$, por dez dias. Apesar da inexistência de evidências de alterações, após dez dias, a análise foi continuada para certificação da esterilidade dos produtos. A análise visou a quantificar os seguintes micro-organismos: clostrídios butíricos incubação, a $30{ }^{\circ} \mathrm{C}$, por dois a cinco dias -, termófilos "flat sour" - incubação, a $55^{\circ} \mathrm{C}$, por dois a cinco dias -
, bactérias láticas - incubação, a $30^{\circ} \mathrm{C}$, por dois a quatro dias - e bolores e leveduras - incubação, a $30^{\circ} \mathrm{C}$, por dois a quatro dias (Apha, 2001; Silva et al. 2010).

As amostras dos três tratamentos foram submetidas a esse tipo de análise durante quatro momentos, sendo o primeiro no tempo zero, logo após o preparo, e os demais a cada 30 dias (30, 60 e 90). Até o momento da análise, as amostras ficaram armazenadas na mesma estufa incubadora para estudo de prazo de validade, a $25{ }^{\circ} \mathrm{C}$.

\section{Análise físico-química}

As análises de $\mathrm{pH}$ e de sólidos solúveis foram realizadas em triplicata, no IT da UFRRJ, no Laboratório de Análise de Carotenoides e Produtos de Origem Vegetal (LACPROV), de acordo com os métodos preconizados pelo Instituto Adolfo Lutz (2008).

Os valores de $\mathrm{pH}$ foram obtidos com $\mathrm{pHmetro}$ de bancada, calibrado com as soluções tampão de pH 4,0 e pH 7,0. A análise do teor de sólidos solúveis foi realizada utilizando-se refratômetro de Abbé, com escala graduada de Brix, previamente calibrado com água destilada a $20{ }^{\circ} \mathrm{C}$. As médias foram comparadas pelo teste de Tukey ( $\mathrm{p}<0,05$ ), utilizando-se o "software" GraphPad Prism, Versão 5 (Graphpad, 2011).

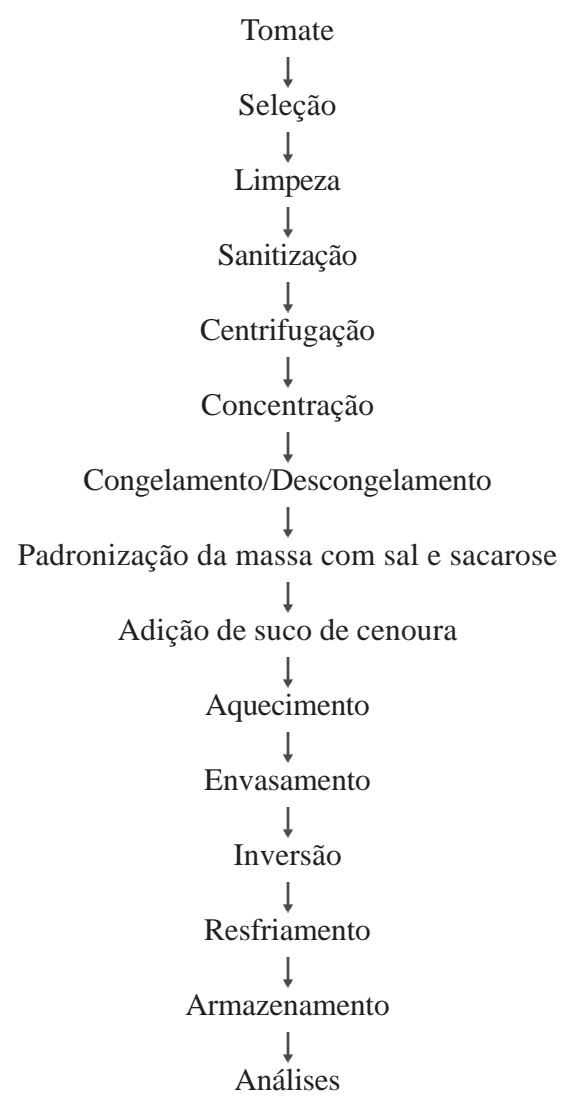

Figura 1. Fluxograma do processamento da massa de tomate com suco de cenoura. 
No acompanhamento da vida útil de todos os produtos, foram feitas a quantificação dos carotenoides e a análise do perfil carotenogênico. A coleta foi feita por amostragem e por tempo estudado, determinados conforme Rodriguez-Amaya (2001), no LACPROV. Para verificação da variação dos resultados ao longo do tempo, foi usado o mesmo software citado anteriormente.

No estudo, foi possível quantificar os teores de licopeno, $\alpha$-caroteno e $\beta$-caroteno das amostras, uma vez que se utilizou como método analítico a cromatografia líquida de alta eficiência (CLAE). Utilizaram-se, ainda, os fatores de conversão de á-caroteno e $\beta$-caroteno, descritos pela Resolução da Diretoria Colegiada n²69 (Brasil, 2005). Os totais de pró-vitamina A foram calculados a partir do somatório em microgramas $(\mu \mathrm{g})$ de retinol dos dois carotenoides pesquisados. Considerou-se $1 \mu \mathrm{g}$ de $\alpha$ caroteno como equivalente a $0,084 \mu \mathrm{g}$ de retinol e $1 \mu \mathrm{g}$ de $\beta$-caroteno, como equivalente a $0,167 \mu \mathrm{g}$ de retinol, conforme preconizado pela legislação vigente.

Para as análises, foi utilizado um cromatógrafo líquido de alta eficiência Waters modelo 2690, equipado com injetor automático e detectores de arranjo de diodos. Todo o sistema foi controlado pelo software Empoyer. A coluna foi um XTerra ODS2, C18 monomérica, $3 \mu \mathrm{m}, 4,6$ x $250 \mathrm{~mm}$. Logo antes da injeção, a amostra foi dissolvida em $2 \mathrm{~mL}$ de acetona e foram injetados $10 \mu \mathrm{L}$. A fase móvel foi um gradiente de acetonitrila:metanol:acetato de etila, contendo $0,05 \%$ de trietilamina, começando com uma proporção de 95:5:0 até 60:20:20 em 20 minutos, permanecendo nesta proporção até o final da corrida. A vazão foi de $0,5 \mathrm{~mL} / \mathrm{min}$ e o reequilíbrio da coluna foi de 15 minutos.

\section{Análise sensorial}

Para a realização da avaliação, o projeto foi, inicialmente, submetido ao Comitê de Ética e Pesquisa da UFRRJ que, posteriormente, informou a sua concordância para execução, por meio do processo de número 23083.010762/2011-16. O método afetivo escolhido para os produtos foi avaliação da aceitação, que é utilizada quando se deseja conhecer o comportamento do consumidor em relação ao produto (Meilgard et al., 1999).

As avaliações ocorreram no laboratório de Análise Sensorial da UFRRJ. Os participantes receberam uma ficha de avaliação da aceitação e foram também questionados sobre nome, idade, sexo, frequência de consumo de massa de tomate e intenção de compra dos produtos.

Os testes foram realizados em cabines, utilizandose luz branca, por 39 indivíduos não treinados, sendo esses professores, graduandos e funcionários do IT da UFRRJ, que avaliaram a aceitação das formulações controle - sem suco de cenoura - e de massa de tomate com três concentrações de suco de cenoura. Foi utiliza- do teste de escala hedônica de 9 pontos (1: desgostei muitíssimo; 2: desgostei muito; 3: desgostei moderadamente; 4: desgostei ligeiramente; 5: nem gostei nem desgostei; 6: gostei ligeiramente; 7: gostei moderadamente; 8: gostei muito; 9: gostei muitíssimo), segundo Meilgard et al. (1999), para os provadores avaliarem cada uma das amostras em relação a sua aparência, cor, aroma, sabor e consistência.

Porções de $30 \mathrm{~g}$ (medida caseira - 2 colheres de sopa) de cada produto foram servidas à temperatura ambiente, em copos brancos descartáveis, codificados com números de três algarismos, juntamente com uma colher. Os julgadores, que não foram informados sobre o teor de suco de cenoura adicionado nas formulações elaboradas, receberam água, também à temperatura ambiente, para limpeza do palato entre a avaliação de uma amostra e outra. Além disso, as amostras foram servidas sem o acompanhamento de outros alimentos.

Os resultados foram avaliados por meio de Análise da Variância (ANOVA) e teste de Tukey, a 5\% de significância, para verificar diferenças significativas entre as amostras, utilizando-se também o "software" GraphPad Prism, Versão 5 (Graphpad, 2011).

\section{RESULTADOS E DISCUSSÃO}

\section{Análise microbiológica}

As três formulações não se alteraram ao longo dos 90 dias, indicando a aprovação dos produtos no teste de esterilidade comercial. A legislação (Brasil, 2001) não determina um limite de tolerância para os micro-organismos analisados, pois esse é um teste qualitativo e o produto não seria comercialmente estéril se o laudo fosse positivo.

O acondicionamento das formulações em embalagens hermeticamente fechadas pode ter contribuído para o resultado satisfatório, assim como o processamento térmico e o resfriamento adequados. Ao analisar microbiologicamente os extratos de tomate desenvolvidos, Pereira (2007) também considerou os produtos comercialmente estéreis.

De acordo com Baglioni (1998), o uso de matériaprima de boa qualidade, a lavagem e a seleção adequadas dessa matéria-prima, a sanitização e a condição asséptica da área de processamento e embalagem são medidas de controle tanto de fungos termorresistentes quanto de fungos comuns na planta de processamento de tomate.

\section{Análise físico-química}

$\mathrm{O}$ pH aumentou de forma significativa após 90 dias de armazenamento, não sendo significativa apenas a variação entre 30 e 60 dias, para as três formulações, como mostra a Tabela $1 \mathrm{~A}$. 
Para Monteiro et al. (2008), é desejável um pH inferior a 4,5 para impedir a proliferação de micro-organismos, pois valores superiores ao $\mathrm{pH} 4,5$ requerem períodos mais longos de processamento térmico da matériaprima, ocasionando maior custo de energia e de processamento.

No trabalho em questão, a partir de 90 dias após o armazenamento, o pH das 3 formulações foi maior que 4,5, sugerindo a interrupção do consumo. Essas variações do $\mathrm{pH}$ podem ser justificadas pelas misturas das matérias-primas principais - tomate e cenoura -, causando alterações pequenas de $\mathrm{pH}$, mas estatisticamente significativas. A proliferação microbiológica foi descartada, por ausência de crescimento nos testes microbiológicos. No entanto, sendo o $\mathrm{pH}$ superior a 4,5 sugere-se o consumo dos produtos em até 60 dias, para uma maior segurança do consumidor.

No estudo de Monteiro (2008), o valor de pH da massa de tomate sem cogumelo oscilou entre 3,59 e 3,82 , não variando significativamente $(\mathrm{p}<0,05)$ ao longo dos seis meses. Também não variou o $\mathrm{pH}$ das duas diferentes formulações de massa de tomate com o cogumelo Agaricus brasiliensis. Os valores de $\mathrm{pH}$ oscilaram entre 3,62 e 3,97, na amostra com $1,4 \%$ de cogumelo, e entre 3,71 e 3,95 , naquela com $3 \%$ de cogumelo.

Com relação ao teor de sólidos solúveis, a Tabela 1B mostra que, ao longo do tempo, não foram constatadas diferenças significativas entre as amostras de massa de tomate com suco de cenoura. Esse fato sugere que as enzimas pécticas, indesejáveis na massa de tomate, foram inativadas durante a concentração e o aquecimento, pois a presença dessas enzimas poderia causar uma diminuição da viscosidade dos produtos, alterando, consequentemente, o teor de sólidos solúveis ao longo do tempo de modo significativo. Monteiro (2008) também não encontrou diferença significativa entre o teor de sólidos solúveis das amostras, ao longo do tempo.
Ao se comparar as Figuras 2 e 3, que tratam do perfil carotenogênico das matérias-primas e do produto, respectivamente, pode-se verificar que foi possível incorporar carotenoide pró-vitamínico A do suco de cenoura, que se apresentava inexistente no concentrado.

A Tabela 2 mostra o teor de carotenoides dos produtos após a adição de 20,30 ou 50\% de suco de cenoura, assim como a degradação dos carotenoides, que foi significativa ao longo do tempo. Segundo Rodriguez-Amaya (2001), o alto grau de insaturação dos carotenoides torna esses compostos susceptíveis à isomerização e à degradação oxidativa. De acordo com Rodriguez-Amaya (1997), a retenção da pró-vitamina Aé favorecida pelas baixas temperaturas, proteção da luz e exclusão do oxigênio.

Neste estudo, os produtos permaneceram armazenados à temperatura ambiente e não em condições de baixa temperatura, o que pode, dentre outros fatores, ter contribuído para a degradação dos carotenoides. A exposição à luz pode ter influenciado, pois os potes de vidro usados para o armazenamento dos produtos eram transparentes. Além disso, a exposição ao oxigênio, durante a análise, pode também ter interferido. Os resultados encontrados corroboraram os de Silva (2008), que observou uma degradação significativa do $\alpha$-caroteno e $\beta$-caroteno, ao longo dos 120 dias de armazenamento do doce de abóbora com cenoura.

Apesar da perda, que já era esperada com a vida útil, o produto apresentou-se com alto conteúdo, mesmo após 90 dias de armazenamento, sendo capaz de atingir pelo menos 30\% da IDR para crianças entre 4 e 6 anos de idade, considerando-se a RDC n ${ }^{\circ} 54$ de 12 de novembro 2012, como mostra a Tabela 3A e B.

Destacando-se a mesma RDC no 54 de 12 de novembro de 2012, os produtos elaborados poderiam também ser nomeados segundo a porção sugerida para consumo, nesse caso, $30 \mathrm{~g}$, ou medida caseira igual a duas colheres de sopa. Sendo assim, das três massas de tomate elaboradas, somente a proporção $80 \%$ de massa de tomate

Tabela 1. Valores de $\mathrm{pH}$ em massa de tomate $(\mathrm{MT})$ com suco de cenoura $(\mathrm{SC})^{1}(\mathbf{A})$ Teores de sólidos solúveis ( $\left.{ }^{\circ} \mathrm{Brix}\right)$ em massa de tomate (MT) com suco de cenoura (SC) ${ }^{1}(\mathbf{B})$

\begin{tabular}{|c|c|c|c|c|c|}
\hline \multicolumn{2}{|c|}{ Tratamentos } & \multicolumn{4}{|c|}{ Períodos de tempo (dias) } \\
\hline MT (\%) & $\mathrm{SC}(\%)$ & Zero & 30 & 60 & 90 \\
\hline 80 & 20 & $4,12 \mathrm{a}$ & $4,31 b$ & $4,31 b$ & $4,63 \mathrm{c}$ \\
\hline 70 & 30 & $4,19 \mathrm{a}$ & $4,32 b$ & $4,33 b$ & $4,65 \mathrm{c}$ \\
\hline 50 & 50 & $4,28 \mathrm{a}$ & $4,44 \mathrm{~b}$ & $4,42 \mathrm{~b}$ & $4,73 \mathrm{c}$ \\
\hline \multicolumn{2}{|c|}{ Tratamentos } & \multicolumn{3}{|c|}{ Períodos de tempo (dias) } & (B) \\
\hline MT (\%) & $\mathrm{SC}(\%)$ & Zero & 30 & 60 & 90 \\
\hline 80 & 20 & $24 \mathrm{a}$ & $22 \mathrm{a}$ & $23 \mathrm{a}$ & $22 \mathrm{a}$ \\
\hline 70 & 30 & $22 \mathrm{a}$ & $21 \mathrm{a}$ & $22 \mathrm{a}$ & $21,2 \mathrm{a}$ \\
\hline 50 & 50 & $17 \mathrm{a}$ & $17 \mathrm{a}$ & $17 \mathrm{a}$ & $17,4 \mathrm{a}$ \\
\hline
\end{tabular}

${ }^{1}$ Letras iguais em uma mesma linha indicam que não há diferença significativa entre as amostras a 5\% de significância. 

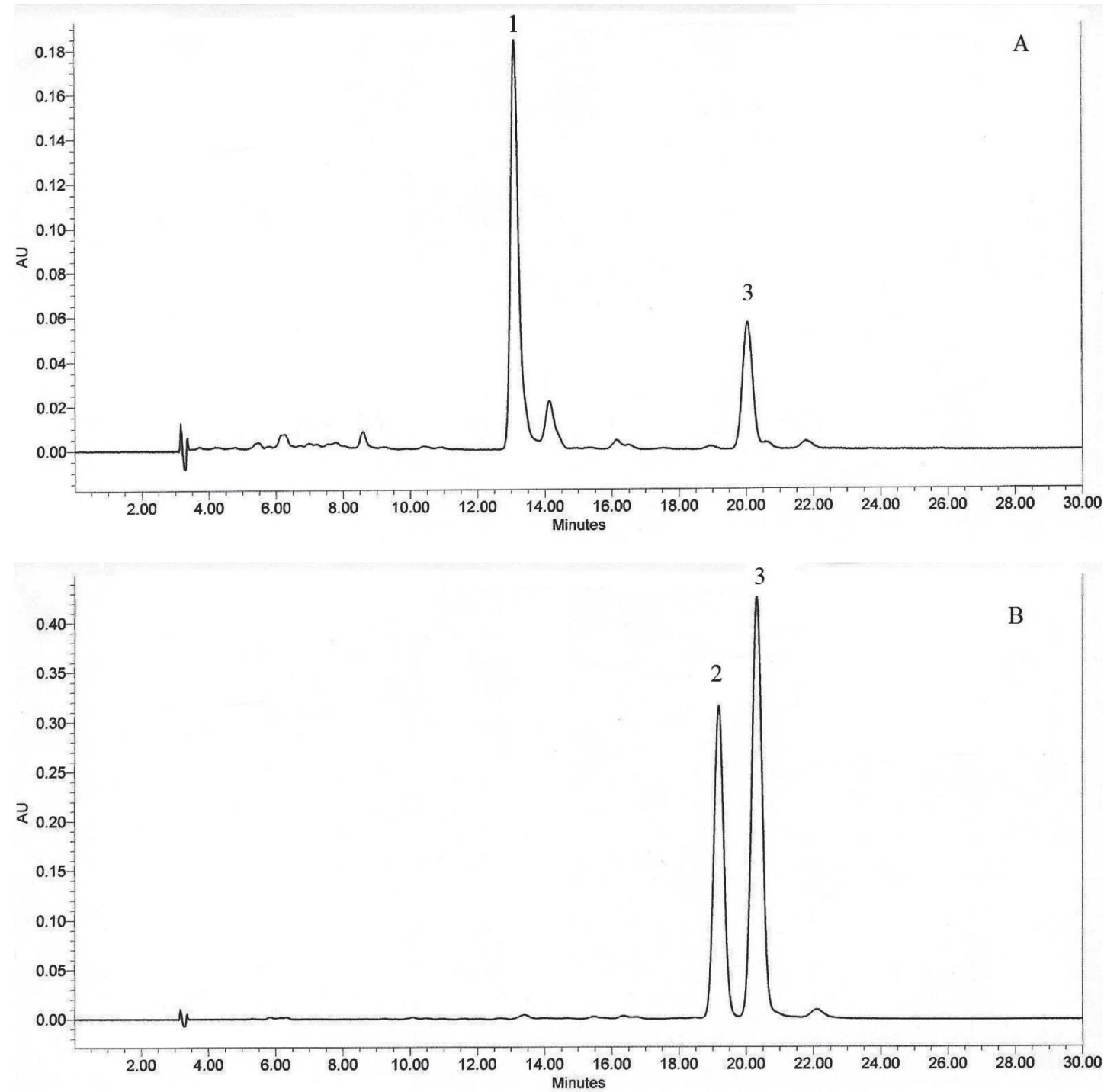

Figura 2. Perfil de carotenoides das matérias-primas usadas na elaboração da massa de tomate com suco de cenoura. Massa de tomate (A); Suco de cenoura. Picos: (1) Licopeno, (2) $\alpha$-caroteno, (3) $\beta$-caroteno, Suco de cenoura (B).

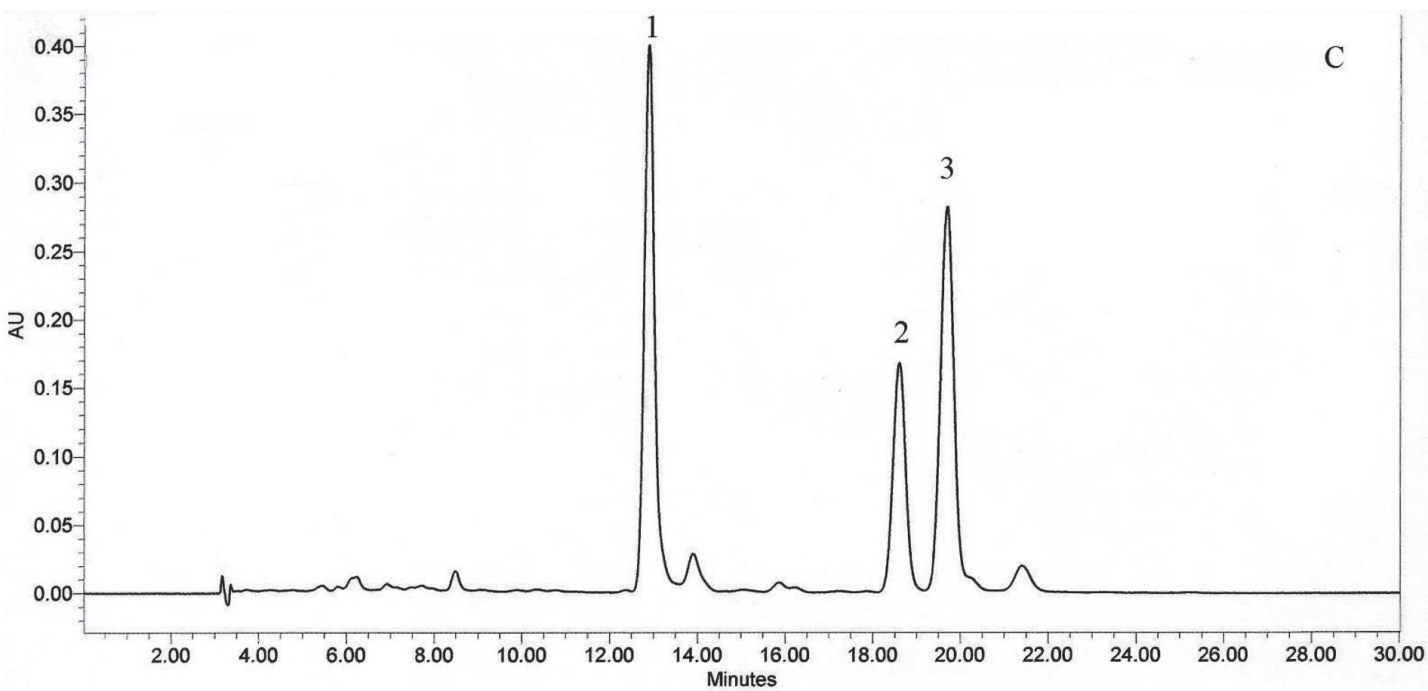

Figura 3. Perfil de carotenoides do produto. Massa de tomate com suco de cenoura.

Picos: (1) Licopeno, (2) $\alpha$-caroteno, (3) $\beta$-caroteno, Massa de tomate com suco de cenoura (C). 
com $20 \%$ de suco de cenoura não receberia a denominação de "fonte". As demais formulações atendem às especificações da citada resolução, conforme apresentado na Tabela 4A e B.

\section{Análise sensorial}

Dentre os 39 entrevistados, com idade compreendida entre 17 e 50 anos, $68 \%$ pertenciam ao sexo feminino e $32 \%$ ao masculino. A faixa etária prevalecente foi entre 17 e 30 anos, somando aproximadamente $90 \%$ da população entrevistada. Com relação ao consumo da massa de tomate, dos $85 \%$ que afirmaram consumir com frequência, $62 \%$ informaram consumir esse produto uma ou duas vezes por semana. Segundo os relatos, os produtos mais consumidos, usando-se massa de tomate, foram massa, "pizza" e carne, nessa ordem, dentre outros em menor escala.

A Tabela 5 mostra que não houve diferença significativa $(\mathrm{p}<0,05)$, entre as amostras, para aceitação dos atributos aparência, aroma e sabor, que, por sua vez, recebeu a menor média de notas, em comparação com os demais, oscilando entre 5,77 e 5,97, que correspondem, na escala hedônica, a nem gostei, nem desgostei.

A cor foi o atributo que apresentou maior média de notas, para a maioria das amostras, oscilando entre 6,31 e 7,41, que compreendem, na escala hedônica, às afetividades de gostar ligeiramente e gostar moderadamente. Para o mesmo atributo, a amostra com 50\% de suco diferiu significativamente das amostras controle e com $20 \%$ de suco. A maior média de notas para a amostra controle esteve, provavelmente, associada à proximidade do produto com a sua apresentação original no mercado, que apresenta coloração vermelha, mais evidente na amostra sem suco.

Outra variação significativa foi verificada no atributo consistência, em que a amostra com $50 \%$ de suco diferiu significativamente de todas as demais. A consistência dessa amostra possivelmente agradou menos aos

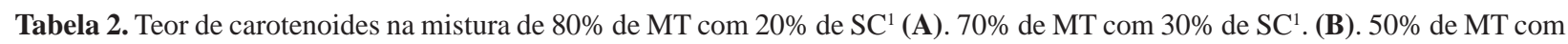
$50 \%$ de $\mathrm{SC}^{1}(\mathbf{C})$

\begin{tabular}{lccc}
\hline Períodos de tempo (dias) & Licopeno $(\boldsymbol{g} / \mathbf{g})$ & $\boldsymbol{\alpha}$-caroteno $(\boldsymbol{\mu g} / \mathbf{g})$ & $\boldsymbol{\beta}$-caroteno $(\boldsymbol{\mu g} / \mathbf{g})$ \\
\hline Zero & $20,5 \mathrm{a}$ & $2,4 \mathrm{a}$ & $21,5 \mathrm{a}$ \\
30 & $13,6 \mathrm{~b}$ & $1,6 \mathrm{~b}$ & $11,7 \mathrm{~b}$ \\
60 & $9,4 \mathrm{c}$ & $1,2 \mathrm{c}$ & $9,7 \mathrm{c}$ \\
90 & $8,7 \mathrm{~d}$ & $1,1 \mathrm{c}$ & $7,6 \mathrm{~d}$ \\
\hline Períodos de tempo (dias) & Licopeno $(\boldsymbol{\mu g} / \mathbf{g}$ & $\boldsymbol{\alpha}$-caroteno $(\boldsymbol{\mu g} / \mathbf{g})$ & $\boldsymbol{\beta}$-caroteno $(\boldsymbol{\mu g} / \mathbf{g})$ (B) \\
\hline Zero & $20,5 \mathrm{a}$ & $7,8 \mathrm{a}$ & $22,7 \mathrm{a}$ \\
30 & $19,3 \mathrm{~b}$ & $6,2 \mathrm{~b}$ & $21,7 \mathrm{~b}$ \\
60 & $18,9 \mathrm{c}$ & $4,3 \mathrm{c}$ & $21,5 \mathrm{~b}$ \\
90 & $7,7 \mathrm{~d}$ & $2,3 \mathrm{~d}$ & $12,5 \mathrm{c}$ \\
\hline Períodos de tempo (dias) & Licopeno $(\boldsymbol{\mu g} / \mathbf{g})$ & $\boldsymbol{\alpha}$-caroteno $(\boldsymbol{\mu g} / \mathbf{g})$ & $\boldsymbol{\beta}$-caroteno $(\boldsymbol{\mu g} / \mathbf{g})$ (C) \\
\hline Zero & $7,1 \mathrm{a}$ & $4,9 \mathrm{a}$ & $19,6 \mathrm{a}$ \\
30 & $5,2 \mathrm{~b}$ & $4,4 \mathrm{~b}$ & $1,1 \mathrm{~b}$ \\
60 & $2,4 \mathrm{c}$ & $4,2 \mathrm{bc}$ & $18,6 \mathrm{c}$ \\
90 & $1,2 \mathrm{~d}$ & $4,0 \mathrm{c}$ & $15,4 \mathrm{~d}$ \\
\hline
\end{tabular}

${ }^{1}$ Letras iguais em uma mesma coluna indicam que não há diferença significativa entre as amostras a 5\% de significância.

Tabela 3. Teor de retinol $(\mu \mathrm{g} / 100 \mathrm{~g})^{1}$ (A). Percentual da IDR atingido para crianças entre 4 e 6 anos de idade ${ }^{2}$ ao longo do tempo (B)

\begin{tabular}{|c|c|c|c|c|c|}
\hline \multicolumn{2}{|c|}{ Tratamentos } & \multicolumn{4}{|c|}{ Períodos de tempo (dias) } \\
\hline MT (\%) & $\mathrm{SC}(\%)$ & Zero & 30 & 60 & 90 \\
\hline 80 & 20 & 379,3 & 208,8 & 172,1 & 136,2 \\
\hline 70 & 30 & 444,6 & 414,5 & 395,2 & 228,1 \\
\hline 50 & 50 & 368,5 & 356,0 & 345,9 & 290,9 \\
\hline \multicolumn{2}{|c|}{ Tratamentos } & \multicolumn{4}{|c|}{ Períodos de tempo (dias) } \\
\hline MT (\%) & $\mathrm{SC}(\%)$ & Zero & 30 & 60 & 90 \\
\hline 80 & 20 & $84,3 \%$ & $46,4 \%$ & $38,2 \%$ & $30,0 \%$ \\
\hline 70 & 30 & $98,8 \%$ & $92,1 \%$ & $87,8 \%$ & $50,7 \%$ \\
\hline 50 & 50 & $81,9 \%$ & $79,1 \%$ & $76,9 \%$ & $64,6 \%$ \\
\hline
\end{tabular}

${ }^{1} 1 \mu \mathrm{g}$ de b-caroteno $=0,167 \mathrm{mg}$ de retinol; $1 \mu \mathrm{g}$ dos demais pró-vitamínicos A= 0,084 mg de retinol.

${ }^{2}$ Considerando a IDR para a faixa etária citada, que é equivalente a $450 \mathrm{mg}$ de retinol. 
Tabela 4. Teor de retinol $\left(\mu \mathrm{g} / 30 \mathrm{~g}^{*}\right)^{1}$ (A). Percentual da IDR atingido para crianças entre 4 e 6 anos de idade ${ }^{2}$ ao longo do tempo (B)

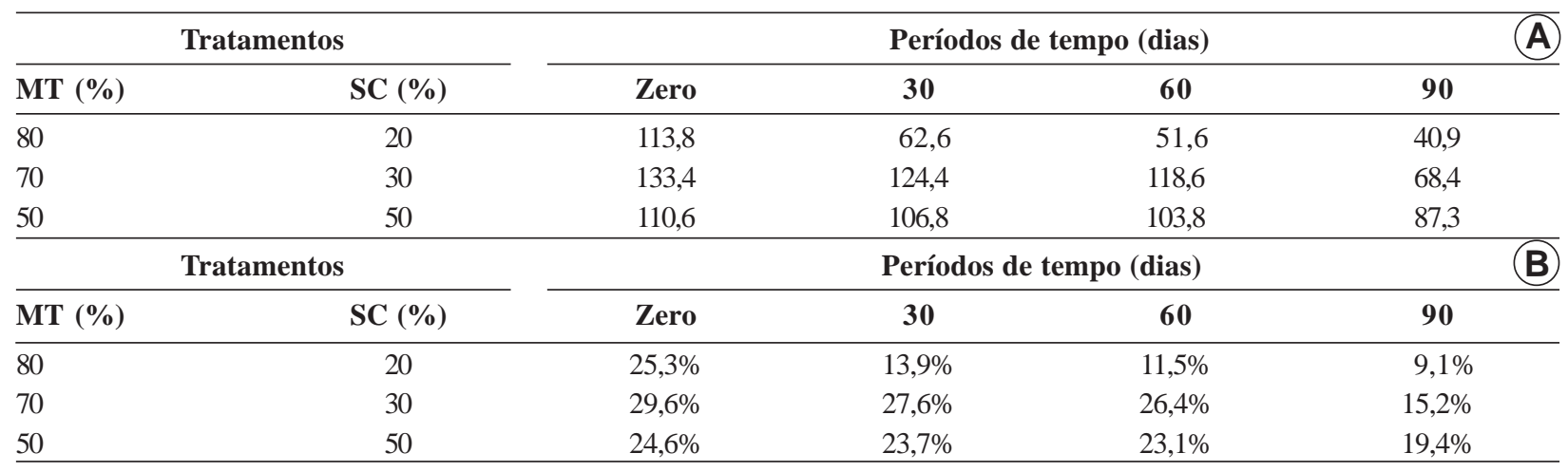

* Considerando $30 \mathrm{~g}$ ou 2 colheres de sopa como porção sugerida para consumo em produtos industrializados.

${ }^{1} 1 \mu \mathrm{g}$ de $\beta$-caroteno $=0,167 \mu \mathrm{g}$ de retinol; $1 \mu \mathrm{g}$ dos demais pró-vitamínicos $\mathrm{A}=0,084 \mu \mathrm{g}$ de retinol.

Tabela 5. Médias de aceitação das amostras de massa de tomate com e sem suco de cenoura ${ }^{1}$

\begin{tabular}{lcccc}
\hline Atributos & Controle & $\mathbf{8 0 \%}$ MT e 20\%SC & $\mathbf{7 0 \%}$ MT e 30\% SC & $\mathbf{5 0 \%}$ MT e 50\%SC \\
\hline Aparência & $6,69 \mathrm{a}$ & $6,90 \mathrm{a}$ & $6,90 \mathrm{a}$ & $6,21 \mathrm{a}$ \\
Cor & $7,41 \mathrm{a}$ & $7,21 \mathrm{a}$ & $7,08 \mathrm{ab}$ & $6,31 \mathrm{~b}$ \\
Aroma & $6,79 \mathrm{a}$ & $6,67 \mathrm{a}$ & $6,46 \mathrm{a}$ & $6,87 \mathrm{a}$ \\
Sabor & $5,77 \mathrm{a}$ & $5,97 \mathrm{a}$ & $5,85 \mathrm{a}$ & $5,82 \mathrm{a}$ \\
Consistência & $6,79 \mathrm{a}$ & $6,92 \mathrm{a}$ & $6,87 \mathrm{a}$ & $5,82 \mathrm{~b}$ \\
\hline
\end{tabular}

${ }^{1}$ Letras iguais em uma mesma linha indicam que não há diferença significativa entre as médias $(\mathrm{p}<0,05)$.

provadores, pela diminuição do teor de sólidos solúveis, que a adição de suco provocou no produto, tornando-o com aspecto de massa mais mole.

Apesar das variações, a literatura mostra que o consumidor costuma aceitar bem produtos adicionados de ingredientes, com o intuito de aumentar o seu valor nutricional. Neste estudo, aproximadamente $62 \%$ dos indivíduos já apresentam o hábito de consumir produtos com esta característica. No doce de abóbora com pró-vitamina A da cenoura, utilizada na forma de cubos, 57,5 e $65 \%$ dos julgadores deram nota máxima para os atributos aparência e cor, respectivamente.

Com relação à intenção de compra, 79\% comprariam uma das amostras com suco de cenoura. Dentre essas, maior parte relatou que compraria a massa de tomate com 50 e com $30 \%$ de suco, apesar da menor apreciação da consistência da amostra com maior adição de suco.

\section{CONCLUSÕES}

Foi possível desenvolver e processar produtos de massa de tomate com suco de cenoura. As condições de processamento dos produtos foram capazes de garantir a segurança microbiológica das formulações, ao longo dos dias, de acordo com o teste de esterilidade para produtos ácidos.

O aumento do conteúdo de carotenoides da massa de tomate com suco de cenoura foi viável, de forma que os produtos alcançaram um teor de retinol por 100 g de amostra de 30 a 64,6\%, considerando-se a percentagem da IDR para crianças entre 4 e 6 anos, no período de 90 dias de armazenamento, à temperatura ambiente, em embalagens hermeticamente fechadas. Analisando-se a porção recomendada de consumo, atingiram valores entre 9,1 e $19,4 \%$, sob as mesmas variáveis. Além disso, os três tratamentos de massa de tomate com suco de cenoura causaram boa impressão sensorial.

Logo, o concentrado de tomate com suco de cenoura, especialmente aquele com $50 \%$ do suco, pode ajudar nos problemas de deficiência de vitamina A, contribuindo para o aumento do retinol sérico em crianças de 4 a 6 anos, comumente afetadas pela hipovitaminose A, considerando-se o teor de carotenoides próvitamínicos A que esse produto agrega. Faz-se necessário estudo de intervenção, de modo a verificar se o consumo do produto estabelece uma estratégia adicional à suplementação por meio da bioconversão dos carotenoides.

\section{REFERÊNCIAS}

APHA - American Public Health Association (2001) Compendium of methods for the microbiological examination of foods. $4^{\mathrm{a}} \mathrm{ed}$. Washington, Editora APHA. 676p.

Baglioni F (1998) Estudo da ocorrência de fungos filamentosos termorresistentes empolpa de tomate envasada assepticamente. Dissertação de Mestrado. Universidade Estadual de Campinas, Campinas. 94p. 
Brasil (2001) Agência Nacional de Vigilância Sanitária. Regulamento técnico sobre padrões microbiológicos para produtos expostos à venda ou de alguma forma destinados ao consumo. Resolução da Diretoria Colegiada ${ }^{\circ} 12$, de 2 de janeiro de 2001. Diário Oficial da União, 10 de janeiro de 2001.

Brasil (2005) Agência Nacional de Vigilância Sanitária. Regulamento técnico sobre a Ingestão Diária Recomendada (IDR) de proteína, vitaminas e minerais. RDC $n^{\circ} 269$. Diário Oficial da União, 23 de setembro de 2005 .

Brasil (2012) Agência Nacional de Vigilância Sanitária. Regulamento técnico sobre informação nutricional complementar. Resolução da Diretoria Colegiada n ${ }^{\circ} 54$, de 12 de novembro de 2012. Diário Oficial da União, 21 de agosto de 2006.

Clinton SK (1998) Lycopene: chemistry, biology, and implications for human health and disease. Nutrition Reviews, 56:35-51.

Geraldo RRC, Paiva SAR, Pitas AMCS, Godoy I \& Campana AO (2003) Distribuição da hipovitaminose A no Brasil nas últimas quatro décadas: ingestão alimentar, sinais clínicos e dados bioquímicos. Revista de Nutrição, 16:443-460.

Graphpad Prism (2011) GraphPad Prism Software. Versão 5. Disponível em: <http://www.graphpad.com>. Acessado em: 08 de dezembro de 2011.

Instituto Adolfo Lutz (2008) Métodos físico-químicos para análise de alimentos. $4^{\text {a } e d . ~ S a ̃ o ~ P a u l o, ~ E d i t o r a ~ I n s t i t u t o ~ A d o l f o ~ L u t z . ~ 1020 p . ~}$

Meilgaard HL, Civille GV \& Carr BT (1999) Sensory evaluation techniques. Boca Raton, CRC Press. 137p.

Milagres RCRM, Nunes LC \& Pinheiro-Sant'ana HM (2007) A deficiência de vitamina A em crianças no Brasil e no mundo. Revista Ciência \& Saúde Coletiva, 12:1253-1266.

Monteiro CS (2008) Desenvolvimento de molho de tomate Lycopersicon esculentum Mill formulado com cogumelo Agaricus brasiliensis. Tese de Doutorado. Universidade Federal do Paraná, Curitiba. 176p.

Monteiro CS, Balbi ME, Miguel OG, Penteado PTPS \& Haracemiv SMC (2008) Qualidade nutricional e antioxidante do tomate "tipo italiano". Alimentos e Nutrição, 19:25-31.

Organização Mundial da Saúde (2009) Global prevalence of vitamin A deficiency in populations at risk 1995-2005. Geneva, WHO. 57p.

Organização Mundial da Saúde (2000) Nutrition for health and development: a global agenda for combating malnutrition. France, WHO. 86p.
Pereira S (2007) Processamento de tomates (Lycopersicom escullentun Mill.), cv. Débora cultivados de forma tradicional e orgânica, para obtenção de extratos. Dissertação de Mestrado. Universidade Rural do Rio de Janeiro, Seropédica. 75 p.

Ramalho RA, Flores H \& Saunders C (2002) Hipovitaminose A no Brasil: um problema de saúde pública. Revista Panamericana de Salud Pública, 12:117-123.

Ramalho A, Padilha P \& Sauders C (2008) Análise crítica de estudos brasileiros sobre deficiência de vitamina A no grupo materno-infantil. Revista Paulista de Pediatria, 26:392-399.

Rodriguez-Amaya DB (2001) A guide to carotenoid analysis in foods. Washington, ILSI Press. 64p.

Rodriguez-Amaya DB (1985) Os carotenóides como precursores de vitamina A. Bol SBCTA, 19:227-242.

Rodriguez-Amaya DB (1997) Carotenoids and food preparation: the retention of provitamin A carotenoids in prepared, processed, and stored foods. EUA, Agency for International Development - OMNI Project. 93p.

Silva EB (2008) Desenvolvimento de produtos alimentares adicionados de ferro, cálcio, zinco e carotenóides (alfacaroteno e betacaroteno) como proposta de alimentos enriquecidos ou fontes destes nutrientes. Dissertação de Mestrado. Universidade Federal Rural do Rio de Janeiro, Seropédica. 127p.

Silva N, Junqueira VCA, Silveira NFA, Taniwaki MH, Santos RFS \& Gomes RAR (2010) Manual de métodos de análise microbiológica de

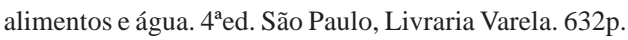

Stahl W, Junghans A, Boer B, Driomina ES, Briviba K \& Sies H (1998) Carotenoid mixtures protect multilamellar liposomes against oxidative damage: synergistic effects of lycopene and lutein. FEBS letters, 427:305-308.

Tawata N (2010) Determinação de carotenóides em alimentos brasileiros in natura processados e preparados para a tabela nacional de composição de alimentos. Dissertação de Mestrado. Universidade Estadual de Campinas, Campinas. 65p.

West CE, Castenmiller JJM (1998) Quantification of "SLAMENGHI" factors for carotenoid bioavailability and bioconversion. International Journal for Vitamin and Nutrition Research, 68:371-377. 